\title{
Le Cheptel cambodgien et son rôle dans l'économie du pays
}

\author{
par R. BARADAT
}

C'

'EST notion banale de parler du cheptel cambodgien comme d'une des ressources principales de ce pays. Symbiose de l'homme et de l'animal tellement profonde qu'il n'est pas de province cambodgienne où la densité du cheptel ne soit en relation directe avec le peuplement humain. Le cheptel occupe une place cardinale dans l'économie interne du Cambodge : façonnage des terres, transport des récoltes, trafic charretier, production de viande indispensable à l'alimentation humaine.

Au delà des frontières du Royaume, le cheptel cambodgien conditionne le ravitaillement en viande des grands centres du Sud-Indochinois. L'avenir, le maintien même de l'immense rizière cochinchoinoise dépendent de la contribution du Cambodge en bétail de trait.

Quelques chiffres tirés d'une approximation annuelle permettront de mesurer le revenu le plus apparent de cette richesse économique.

Bœufs 45.000 abattus au Cambodge 40.000 exportés

$\begin{array}{lllll}\text { Buffles } 2.000 & - & 10.000 & - \\ \text { Porcs } 400.000 & - & 50.000 & - \\ \text { Peaux } 90.000 \text { façonnées ou non. } & \end{array}$

Ce qui représente une infinité de transactions; en multiplier le volume par les prix actuels serait jongler avec des millions de piastres.

Par ses terres siliceuses, latérisées, sols de savanes et de forêts claires, le Cambodge, pour le plus clair de son étendue, est à vocation pastorale et le cheptel y conditionne toute tentative d'amélioration du sol ainsi que tout progrès agricole.

De ce que la rente du sol y est nulle et les soins de l'éleveur réduits à l'extrême, on aurait tort de conclure que c'est là une solide richesse năturelle. Rien de plus instable que la richesse représentée par un troupeau. Les régressions sont très rapides en matière d'élevage; les dommages par épizooties. maladresse ou négligence de gestion blessent à vif, et pour longtemps, les éléments mêmes de restauration.

\section{LES BESOINS \\ DE L'ÉCONOMIE CAMBODGIENNE}

$1^{\circ}$ Besoins en attelages. - L'irrégularité des chutes de pluies, la faible capacité de rétention des sols peu humifères rendent aléatoires des façonnages élémentaires tels que ceux de la rizière. Les labours, les repiquages sont contrariés par l'absence des pluies ou bien des sécheresses tardiyes obligent à des reprises partielles des travaux agraires. Chaque année, une partie dos rizic̀res ne peut être mise en culture, pour des raisons d'ordre climatique beaucoup plus que par manque d'attelages, ceux-ci étant assez régulièrement proportionnés à la maind'œuvre paysanne.

$\mathrm{Si}$ on établit par province la répartition des surfaces cultivables, des attelages nécessaires à ces travaux et de l'excédent de bétail disponible, on remarque l'accord existant entre le chiffre du cheptel et l'importance des cultures. Rien ne saurait mieux traduire la vocation du cheptel cambodgien.

On admet que les rizières - déclarées ou non occupent, au Cambodge, une superficie qui n'est pas inférieure à $1.000 .000 \mathrm{~d}$ 'hectares (Le chiffre qui ressort des estimations cadastrales est de 870.000 hectares). Les hasards du climat font que la superficie totale ne peut jamais être mise en culture, les variations pouvant s'inscrire entre 600.000 hectares et 900.000 hectares. Le rendement est inégal - de 600 à 1.100 kilogrammes de paddy à l'hectare - si bien que la production peut varier du simple au double, de 500.000 à 1.000 .000 de tonnes selon les années.

On admet que le façonnage moyen de la rizière par attelage (bceufs ou buffles) n'est pas supérieur à 4 hectares. Le morcellement des rizières qui, pour une même exploitation paysanne, figurent sous le nom des divers membres de la famille, ne facilite pas les investigations. On en a déduit toutefois que la propriété moyenne en rizières approche de 4 hectares par famille. On ne sera donc pas loin de la vérité en affirmant que 220.000 paires de bœufs ou 


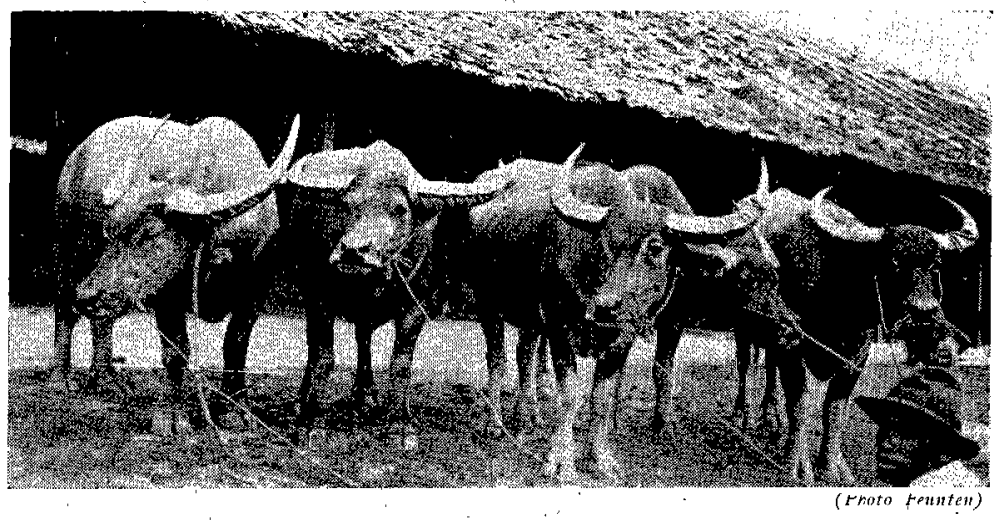

Groupe de buffles cembodgiens modèle courant exportation. 'retient d'autre part un millier. d'attelages.

On peut négliger la vidange. des divers produits forestiers (huile de bois, bambous, ro'tins, résines, gomme-gutte). Ces travaux étant épisodiques et pouvant relever d'attelages distraits pour, un temps des travaux du sol. Il 'n'en reste pas moins que l'exploitation forestiere exige un minimum de 6.000 attelages.

Ainsi, les besoins de bétail de trait peuvent être grossièrement chiffrés :

buffles sont, au Cambodge, annuellement utilisés aux travaux de la rizière.

A cela, il faut 'ajouter les cultures vivrières : 230.000 hectares pour le milis, 30.000 hectares. pour les haricots. Ces cultures, localisées sur les terres de berge, réclament une préparation du sol consistant surtout en labours successifs et hersages jusqu'a pulverisage complet. Les soins accordés à ces façonnages varient selon les régions, en raison de la diversité des superficies par propriétaire. Cette' superficie moyenne est de 2 hectares pour Kandal, Kompong-Cham et Prey-Véng, négligeable pour lés autres provinces, sauf Kratié où elle est de 5 hectares, mais où la mise en état du sol ne peut être portée au delà de 4 hectares. Ainsi, dans les Sroks de Kratié et Chalong, la superficie des cultures vivrières est deux fois plus élevée que celle. réservée aux rizières (10.000 hectares contre 5.000 hectares). 110.000 paires' de bœufs et buffles sont utilisées aux cultures de maiss, haricots et soja.

Dans ce décompte; sont volontairement négligées les cultures de coton, tabac, arachide, ricin, mûrier, canne à sucre... Elles occupent des surfaces relativement réduites. N'ont pas à figurer non plus les cxigcnces d'attclage pour los transports de produits agricoles, du sucre de palme, des produits de l'artisanat rural (vannerie, poterie). Ces travaux, la plupart des charrois retiennent épisodiquement des attelages, mais ne les détournent pas des cultures principales : paddy et maïs.

Néanmoins, il est des attelages qui ont une destination presque exclusive, ceux des entreprises forestières. Pour une exploitation annuelle de 100.000 mètres cubes de bois d'œuvre, on admet qu'il faut 500 triqueballes dont chacun effectue en moyenne 100 voyages avec 2 mètres cubes, l'attelage moyen étant de 10 paires de buffles.

L'exploitation de bois de feu et du charbon de bois

\subsection{0 paires (rizières). \\ 110.000 -. (cultures vivrières). \\ $6.000-$ (exploitation forestière).}

Par contre, les recensements accusent l'existence de 316.000 paires de bcufs, buffles ou bufflèsses adultes, ce qui signifierait, un manque d'attelages. C'est là une contradiction théorique tenant à cè que l'alternance des cultures permet un roulement des attelages difficile à mesurer et dont le mode de. calcul ne peut tenir compte. Divergence apparente venant aussi de ce que les recensements du bétail sont généralement au-dessous de la réalité.

On doit adimettre qu'il y a suffisamment d'attelages pour servir les exigences de l'économie agricole et forestière du. Cambodge. L'inverse, d'ailleurs, serait étonnant si l'on songe à la modération du Cambodgien dans l'emploi de son bétail, modération

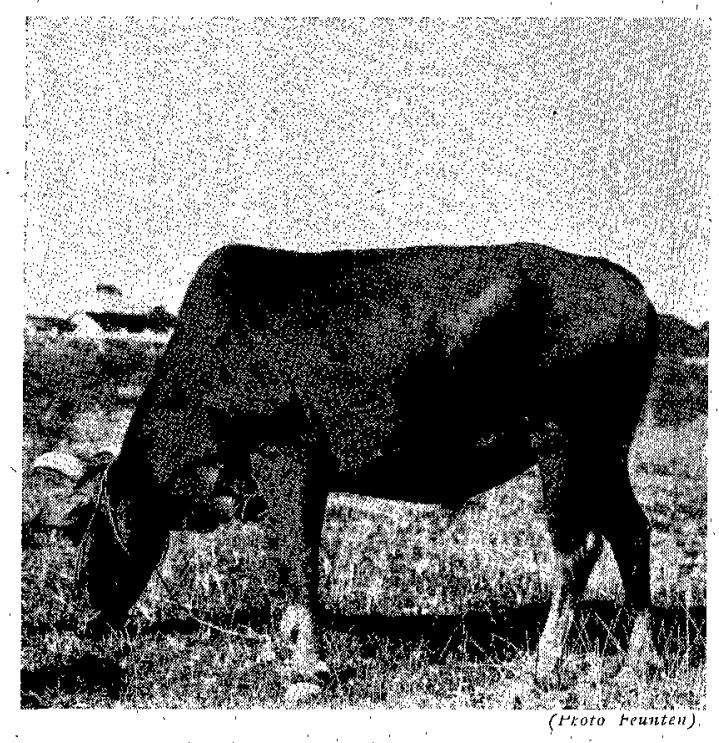

Bouf cambodgien, type ordinaire boucherie. 
qui garde les animaux d'une usure prématurée et de déchéances accentuées par les disettes saisonnières.

Une attraction est exercée par les terres de berge sur les attelages disponibles dans les provinces voisines. Il est indispensable à l'économie du Cambodge que des animaux, nés et grandis dans les savanes boisées, où ils ne sauraient être utilisés efficacement, soient dirigés sur les terres opulentes, riveraines du Mékong et du Bassac. Ils y subissent des modifications de format, sous l'effet d'améliorations alimentaires liées à l'excellence de pâturages qu'on a qualifiés d'herbages d'embouche. La mise en qulture des terres de berge ne peut être réalisée que par l'apport de bétail provenant des provinces voisines. L'utilisation de ce bétail n'entrave pas sa mise en condition et son engraissement.

Dans cet acheminement vers les terres de berge, il est des étapes nécessaires, le jeune bœuf ou buffle turbulent, mal dompté, issu de troupeaux à demi sauvages devant être assagi au cours de deux ou trois saisons culturales en pays de rizière. La physique du sol explique circulation du bétail et transactions d'une province à l'autre, transactions qui. hormis épizooties, doivent rester libres d'une ingérence administrative qui aurait tôt fait d'apparaître tracassière.

Le cheptel du Cambodge, suffisant pour ses besoins

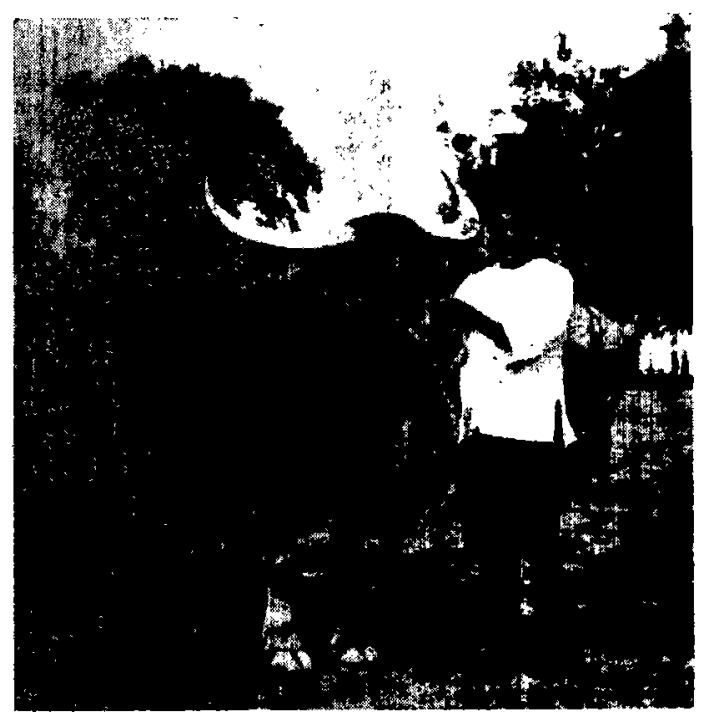

Buffle cambodgien, animal castre en très bon état d'entretien.

actuels, ne pourrait faire face à une extension brusque des cultures. Au demeurant, les possibilités des attelages sont supérieures à celles de la main-d'œuvre locale. On peut donc considérer que, sauf immigration massive de paysans étrangers, le véritable problème agricole ne réside pas tant dans la possibilité de nouveaux défrichements que dans une amélioration des façons culturales et des rendements, amélioration qui, pour une bonne part, reste lièe à l'entretien d'un nombreux bétail et à la production de fumier. S'en tenir aux facilités de travail que procure un cheptel nombreux, maintenir le stade agricole actuel suppose la complète sauvegarde du cheptel. Aller au delà, exige une politique de l'élevage cohérente. Elle s'impose.

$2^{\circ}$ Besoins en viandes. - En exceptant PhnomPenh où le poids moyen des sujets sacrifiés à l'abattoir est supérieur à la moyenne générale, il est abattu annuellement 1 porc pour 10 habitants et 1 boeuf pour 75 habitants. Soit en moyenne 4 kilogrammes de viande de porc et 1 kilogramme de viande de bouf par habitant et par an. Cette consommation va en augmentant, à la mesure de l'accroissement du bien-être général. On serait tenté de trouver très faible cet apport azoté dans la ration, si on la rapporte, par exemple, à celle des habitants des Etats-Unis : environ 80 kilogrammes de viande par personne et par an, dont la moitié est représentée par viande de poro et saindoux. En réalité, les éléments protéiques les plus courants viennent de la consommation de poisson, de volaille, des produits de la chasse ou même de la viande d'animaux morts à la suite de maladies banales. Néanmoins, il est souhaitable que la participation des viandes fraîches dans l'alimentation des Cambodgiens aille en croissant. Dans un raccourci qu'il estimait peu correct mais qui est fort juste, Ch. Richet disait qu' « un peuple colonial a la mortalité de son alimentation $"$. Parmi les populations de la Fédération Indochinoise, 
le Cambodgien se classe en bon rang pour.la consommation de viandes fraîches. Cette consommation peut être ainsi mesurée :

\begin{tabular}{|c|c|c|c|}
\hline & PHNOM-PENH & $\begin{array}{l}\text { CHEFS-LIEUX } \\
\text { provinciaux } \\
\text { etgr.bourgs }\end{array}$ & $\begin{array}{l}\text { ABATAGES } \\
\text { animaux } \\
\text { non controlés }\end{array}$ \\
\hline Boeufs, abattus. & 6.000 & 8.000 & 40.000 \\
\hline Poids 'total. . & $750 \mathrm{T.}$ & $750 \mathrm{~T}$. & $2.000 \mathrm{~T}$. \\
\hline Porcs abattus. & 30.000 & 110.000 & 250.000 \\
\hline Poids total .. & $4.000 \mathrm{~T}$. & $4.000 \mathrm{~T}$ & 7.500 T. \\
\hline
\end{tabular}

Si les rations de viande étaient uniformément réparties, chaque habitant ne consommerait que 20 grammes de viande par jour, dont environ les quatre cinquièmes en viande de porc.'Si l'on admet comme désirable un enrichissement de la ration propre à permettre à chacun un accroissement du rendement physique et intellectuel, on s'aperçoit que le cheptel du Cambodge serait incapable de faire face à une consommation que l'on doit considérer comme normale, celle qui porterait la ration moyenne en viande de 20 grammes à 100 grammes par jour. "On ne peut compter que dans une certaine mesure, avec le régime d'élevage, sur une autorégulation de l'offre et de la 'demande. L'élevage primitif, pastoral ou familial, dont la consommation est encore actuellement presque exclusivement tributaire, est caractérisé par un manque absolu d'organisation, de méthode et aussi par la politique du moindre effort sanctionnée par un rendement minimum. Sur ce terrain, toute tentative d'organisation rencontrerait des obstacles, sans doute connus, mais presque insurmontables 》 (Le Louet, "Consommation du bétail en 'Indochine », Bulletin Economique, Décembre 1931).

A Phnom-Penh, au cours des années 1925 à 1945, le nombre des bœufs abattus a varié entre 4.147 et 7.200, chiffres extrêmes, avec une moyenne, par tête, de 135 kilogrammes; le nombre des porcs a varié de 42.810 à 87.606 , avec un poids moyen de viande de 34 à 49 kilogrammes. La proportion de viande de bceuf a varié de 14 à $36 \%$ de la consommation totale.

Dans les petits villages où n'existent pas de bouchers chinois, la proportion de viande consommée est beaucoup moins élevée. On y sacrifie les bovins accidentés, les malades dont la guérison est improbable, les pré-agoniques et aussi les vaches et les génisses dont les paysans font peu de cas. Ces abatages que ne devrait pas influencer l'esprit de lucre, qui sont directement contrôlés par les intéréssés eux-mêmes, assurent l'utilisation d'un bétail pour lequel les abattoirs sont fermés. L'incon- vénient est qu'ils échappent à tout contrôle et que les éleveurs ne se soucient guère de porter leur choix sur les femelles infécondes ou dignes d'être réformées pour vieillesse ou' affections organiques.

A la suite d'une enquête prolongée dans les tanneries de Phnom-Penh qui récoltent la plupart des peaux du Cambodge, on peut établir l'approximation suivante pour ces abatages (centres provinciaux et villages) :

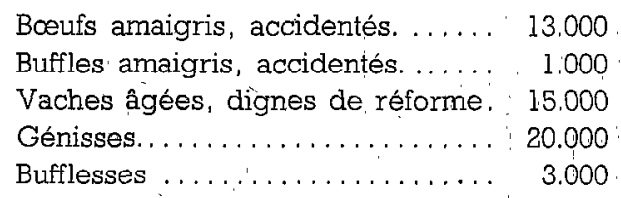

Le nombre des génisses abattues est exagéré, hors de proportion avec les soucis d'une sélection élémentaire. Le cours élevé de la vachette pour la préparatión du box-calf a enlevé de son efficacité à l'interdiction d'abatage des femelles. Cette mesure ne saurait d'ailleurs être absolıe. Les éleveurs, eux-mêmes, devraient être bien placés pour juger de l'opportunité à se débarrasşer des femelles infécondes qui encombrent inutilement les pâturages. L'insouciance des lendemains est; hélas, telle que beaucoup de paysans consentent plutôt à l'abatage d'une génisse qu'à celui d'une vache dont les gestations s'espacent el dont la vie utérine 'est ralentie par l'âge. Ici, intervient la commisération bouddhique pour la vache que l'on voit suitée ou gravide à un stade avancé, alors que le sort de la génisse est' moins attendrissant. En 1941-42, la hausse du prix des peaux, la possibilité exclusive de fabrication. de box-calf à partir des petites peaux ont provoqué une recrudescence des abatages de génisses.

\section{LES RESSOURCES}

Les ressources offertes par le cheptel cambodgien. sont plus ou moins fidelement figurées par les chiffres $\mathrm{du}$ recensement. Le tableau ci-après donne les chiffres officiels de 1915 à 1945.

Ce tableau appelle diverses remarques et réserves.

Il faut observer d'abord la tendance à peu près constante, chez les paysans, à sous-estimer l'importance du troupeau et les profits qu'il peut procurer. D'autre part, les recensements ontrarement été basés sur des considérations sérieuses. Quand des sondages systématiques sont pratiqués, par exemple à l'occasion de vaccinations, on trouve toujours un. chiffre bien supérieur à celui qui est admis par la. statistique officielle.

Parmi les recensements successifs, deux statistiques s'écartent de la ligne générale et doivent être tenues pour fantaisistes. Celle de 1915 qui indique un cheptel nettement en dessous du réel et celle de 1932 qui accuse un gonflement insolite que viennent 
TOTAL DES RECENSEMENTS SUCCESSIFS

\begin{tabular}{|r|c|c|c|c|c|c|c|c|}
\hline & 1915 & 1921 & 1928 & 1932 & 1939 & 1941 & 1943 & 1945 \\
\cline { 2 - 6 } & & & & & \\
Bovins..... & 582.439 & 826.919 & 804.500 & 912.500 & 772.890 & 871.767 & 855.625 & 914.916 \\
Bubalins ... & 366.333 & 550.059 & 519.050 & 804.700 & 338.299 & 325.513 & 313.798 & 327.102 \\
\hline Total... & 948.772 & 1.376 .978 & 1.323 .550 & 1.717 .200 & 1.111 .189 & 1.197 .280 & 1.169 .423 & 1.242 .018 \\
\hline
\end{tabular}

démentir les statistiques immédiatement voisines.

Par contre, la statistique de 1921 qui résulte d'inventaires des ressources du Cambodge au lendemain de la guerre et les statistiques depuis 1939 , découlant d'investigations plus poussées, peuvent être tenues pour recevables et de valeur comparable.

\section{Diminution progressive du cheptel bubalin. -} Cette diminution est manifeste. De 1920 à 1940, sur une période de vingt années, elle représente une déchéance d'environ $40 \%$. Ce parallèle alarmant contient une grande part de vérité : fragilité de ce bétail devant la peste bovine, sensibilité à divers parasitismes, désaffection des éleveurs à mesure que les défrichements gagnent sur la forêt, expliquent le recul de ce cheptel. Le peu de résistance des jeunes bufflons aux conditions sévères de la saison sèche ont fait délaisser cet élevage sur les chiches pâtures des terrains sablonneux ou latérisés. L'espèce étant d'ailleurs tardive, les pâturages restreints sont rapidement encombrés par le nombre des sujets de remplacement qu'il faut mener à l'âge adulte. Le recul de l'élevage des buffles et l'extension parallèle de l'élevage des bœufs peuvent être illustrés par des chiffres empruntés à la province de Kompong-Thom. On y comptait en $1921: 27.137$ bovins et 70.573 bubalins qui sont devenus 60.941 bovins et 40.234 bubalins en 1945. La disparition du buffle est à rapprocher de l'effondrement brusque de l'élevage équin, le nombre des chevaux passant de 40.000 à 4.000 au cours des vingt dernières années. par suite de la négligence des éleveurs et des ravages du surra accentué par l'abandon des règles d'une hygiène élémentaire. Or, le surra prend également chez les buffles, lorsqu'ils sont sousalimentés, l'allure de véritables enzooties. Il serait téméraire de vouloir, dans ce rapprochement, rechercher une marque de l'adaptation du trypanosome d'Evans et un présage de développement du surra comme maladie d'avenir chez le buffle. Toutefois, cette indication de la statistique permet de mesurer la gravité de la crise de l'élevage du buffle, la disparition de cet animal étant fâcheuse, car il est un moteur indispensable à nombre d'entreprises agricoles et forestières. "Il n'y, a pas lieu d'envisager sa disparition avant longtemps et son remplacement rapide par un autre moteur animal ou mécanique. On notera, pour s'en convaincre, qu'il est encore employé aujourd 'hui dans plusieurs États d'Europe (Italie, Balkans). " (Jacotot_L'élevage en Indochine - Indochine, 5 Novembre 1942.)

La capacité digestive du buffle, son pouvoir d'assimilation lui permettent d'utiliser des fourrages grossiers et ligneux, l'aigre et coriace végétation des sols acides, des cuvettes marécageuses, des pâtures inondées. Au plein de la saison sèche, quand l'herbage est pauvre, raréfié, en touffes déchaussées, cet animal, craignant moins les fauves, s'enfonce dans la jungle à la rencontre des bourbiers, des coulées humides et ne dédaigne pas les pousses arbustives rêches.

Grâce à ces particularités, cette espèce peut vivre là où l'entretien du bœuf est plus malaisé.

On sait, par ailleurs, que le recul marqué par l'élevage du buffle eût été moindre avec une conception plus saine des échanges internationaux et une protection plus efficace des droits des éleveurs.

Déséquilibre du cheptel. - A ne considérer que son chiffre global, on est tenté de voir dans le cheptel du Cambodge une richesse naturelle résultant de l'action commune de l'homme et du milieu, selon un automatisme que l'on suppose indéréglable. On est porté à croire que le nombre même suffit à pallier les ravages des épizooties les plus sensationnelles. Or, ce cheptel est" partiellement déséquilibrć et instable par essence, la proportion des femelles étant trop faible pour permettre une restauration ou une reconstitution rapides. Les recensements indiquent les pourcentages ci-aprè : bœufs $45 \%$, vaches $25 \%$, jeunes produits $30 \%$. La disproportion réelle est un peu moins sévère pour le troupeau d'élevage.

Cependant, le Cambodge n'échappe pas à la règle commune aux élevages tropicaux où l'objet essentiel 
est lá produclion du travail, celles de la viande ou du lait restant secondaires. Ici, comme dans l'Inde, les boufs et buffles adultes forment environ $35 \%$ de l'effectif total.

Une confrontation de la statistique avec celle d'un cheptel d'Europe est instructive :'

\begin{tabular}{|c|c|c|}
\hline . : & CAMBODGE & $\begin{array}{l}\text { EUROPE } \\
\text { occident. }\end{array}$ \\
\hline Vaches reproductrices. . . . . . & $25 \%$ & $50 \%$ \\
\hline Taureaux,$\ldots . . . \ldots$. & $0,5 \%$ & $1 \%$ \\
\hline Bœufs ........ & $45 \%$ & $10 \%$ \\
\hline Taurilions, bouvillons et veaux & $16 \%$ & $17 \%$ \\
\hline Génisses, taures et vèles . . . & $13,5 \%$ & $22 \%$ \\
\hline
\end{tabular}

Le faible rendement du bétail cambodgien, son manque de précocité, son inaptitude à la production laitière interdisent d'envisager une orientation. comparable de l'élevage. Le seul revenu possible, pour longtemps encore, est dans l'utilisation des adultes pour divers travaux et dans la vente des sujets d'âge réformés.

Cette confrontation permet de mieux saisir pourquoi l'augmentation numérique du cheptel ne peut être envisagée selon les données classiques en Europe. L'effectif bovin qui, en France, était de 12.500.000 en 1919, passe à 15.200 .000 en 1929, soit un accroissement de $25 \%$ en dix ans, réussite laissée d'ailleurs à la libre initiative des éleveurs.

Au Maroc, la progression du troupeau bovin a été la réussite la plus éclatante des débuts de l'occupation française, l'effectif ayant doublé en l'espace de six ans. I'interdiction de l'abatage des femelles de moins' de 8 ans et des élèves n'ayant pas encore quatre dents de remplacement ont assuré l'augmentation de la quantite du cheptel.

Voici cette progression

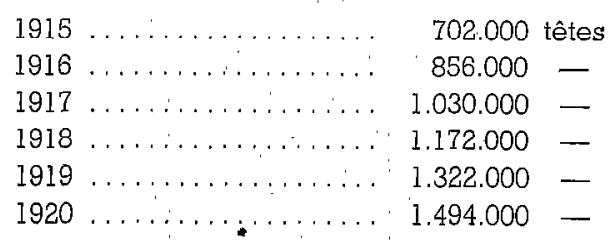

En Australie, le cheptel bovin de 8.491.428 en 1902 , passe à 14.142.400 en 1943 .

Pour obtenir, au Cambodge, un accroissement du cheptél même fort modeste en comparaison de ces chiffres, il faut une politique ferme de l'élevage.

L'espèce bubaline est, elle aussi, susceptible d'accroissement rapide dans des conditions clémentes du milieu. La meilleure illustration de cette faculté me semble être le devenir d'un troupeau de
6 buffles lâchés en 1828 dans l'île Melville par des soldats australiens qui les avaient importés de' Timor. De là, le troupeau gagna le continent australien et essaima à l'est de Port Darwin dans la région' marécageuse de Coburg Péninsula. Ces buffles, vivant à l'état sauvage, se sont tellement multipliés que, de 1926 à 1937, l'abatage au fusil des taureaux, jugés excédentaires, a produit environ 150.000 peaux.

La suite de cette étude montrera les obstacles et les causes d'échec inhérents à un programme d'accroissement du nombre des femelles adultes qui, seul, est significatif d'une augmentatioh de la production et de' l'excédent exportable.

La lecture des statistiques montre qu'un résultat partiel aurait été atteint, le nombre des'vaches passant de 220.000 en 1941 à 329.000 en'! 1945، soit une augmentation do $8 \%$ en cinq années. 'Dans la condition attardée de l'élevage autochtone, 'il faut doser l'importance du troupeau reproducteur en fonction des ressources fourragères et de l'étendue des pâturages. Telle a été la première tranche du programmé suivi depuis 1938 : gestion globale du cheptel simplifiée en raison de son déséquilibre, toutes les possibilités du troupeau reproducteur devant être utilisées à son accroissement. Les progrès obtenus devraient permettre désormais plus de souplesse et, notamment, d'aborder la' deuxième étape par un tri de la production qui reste à la base de toute sélection.

Répartition des sexes, - A lire les statistiques, les jeunes produits âgés de moins de 3 ans formeraient environ $30 \%$ du troupeau.

$$
\begin{aligned}
& \text { En } 1941: 29,4 \% \text { du troupeau bovin } \\
& 26,6 \% \text { - bubalin } \\
& \text { En } 1943: 29,9 \% \quad \div \quad \text { bovin } \\
& 30,8 \% \quad-\quad \text { bubalin } \\
& \text { En } 1945: 32,5 \% \quad-., \text { bovin } \\
& 31 \% \div \text { bubalin }
\end{aligned}
$$

Il nous plait de retrouver dans cette indication de la statistique, une tendance au rajeunissement du cheptel et une promesse pour son avenir. Néanmoins, on peut penser que ces chiffres sont encore inférieurs à la réalité et que les jeunes de moins de 3 ans forment $40 \%$ de l'ensemble du cheptel.

Ce qui froisse davantage, dans tous les recensements, c'est l'inégalité en nombre des jeunes mâles et des jeunes femelles, les mâles étant én majorité, tant sur les bocufs quo sur les buffles. En dépit du scepticisme avec lequel on doit accueillir les évaluations de l'effectif, j'analyserai cette disparité.

En 1941, il y a 56 bouvillons contre 44 génisses

$$
\begin{aligned}
1945,-55-4 \text { bufflons } & -45 \text { - } 51 \text { bufflonnes } \\
\text { En } 1941,-52- & -48
\end{aligned}
$$


Il est d'observation courante, en tous pays, qu'il naît sensiblement autant de produits femelles que de produits mâles. Les facteurs qui régissent le déterminisme du sexe sont assez mal connus et complexes. Nos investigations à l'occasion des concours itinérants, ont souligné de faibles variations. Dans une même série de villages, on observe $48 \%$ de naissances mâles en $1941,52 \%$ en 1942 , $51 \%$ en 1943, l'égalité approximative des sexes apparaissant sur l'ensemble des naissances de trois années.

Je n'ai pu observer une influence de l'âge du géniteur mâle. Celui-ci étant le plus souvent un taurillon, moins âgé que les femelles qu'il féconde, il devrait s'ensuivre une plus forte proportion de. naissances femelles : il n'en est rien.

Je n'ai pas davantage observé une plus grande proportion de naissances mâles sur pâtures médiocres, là où le taureau se trouve sous-alimenté à l'époque principale du rut.

Après le passage d'une grave épizootie de peste bovine dans la province de Pursat en 1936, il a été observé $55 \%$ de naissances femelles sur les vaches et $63 \%$ sur.les bufflesses du Srok de Krakor sévèrement éprouvé. On ne saurait généraliser cette observation unique et $\mathrm{y}$ trouver un témoignage en faveur d'un processus naturel d'auto-défense pour la reconstitution rapide du cheptel.

Malgré quelques contradictions, on pcut dégager une tendance générale dans la répartition des sexes. Sur savane boisée avec points d'eau abondants où les troupeaux retrouvent des conditions identiques à celles des bovidés sauvages, l'égalité des sexes est presque rigoureuse. Sur parcours pauvres, le sexe femelle parait l'emporter parmi les naissances groupées de Janvier à Mars qui font suite à la fécondation de la plus grande partie du troupeau. Sur terres opulentes des berges, les naissances mâles l'emportent nettement, tant en raison des conditions mésologiques, que parce que les éleveurs s'attachent de préférence aux vaches dont naissent des mâles.

En effet, les éleveurs n'accordent pas au produit femelle la même valeur qu'au mâle, le prix du bœuf futur étant deux à trois fois supérieur à celui de la vache adulte, inutilisée aux travaux agricoles. Aussi, la mortalité observée sur les jeunes est généralement plus lourde chez les femelles, objet de soins moins attentifs. Cette disposition d'esprit jointe à la propension à abattre des génisses suffit à expliquer la disparité des sexes parmi les jeunes produits.

- Autrement que la vache, la bufflesse conserve une valeur motrice; aussi, dans cette espèce, n'existe$t$-il pas de défaveur pour les jeunes femelles et les recensements accusent un meilleur équitibre des sexes.
Fécondité des femelles. - Mortalité des jeunes (V. cette Revue, III, 30).

Jennesse du troupeau. - Le cheptel étant peu précoce, le troupeau s'alourdit de la production échelonnée au cours des années nécessaires pour parvenir à l'état adulte ( 4 ans pour la vache et 5 ans pour la bufflesse). Il en résulte un encombrement du pâturage par des animaux que les éleveurs sont tentés de juger improductifs, ce qui les incline à l'abatage des génisses et ce qui, en beaucoup de régions, limite l'accroissement du troupeau de vaches reproductrices.

$\mathrm{Si}$ l'on confronte les chiffres que nous avons recueillis au cours de nos tournées avec ceux que les auteurs classiques indiquent pour le bétail de remplacement, on note que la situation est satisfaisante pour les bovins malgré les abatages de génisses, mais que l'avenir de l'élevage du buffle est compromis, en raison d'un manque de précocité, d'une fécondité plus faible et d'une fragilité plus grande de cette espece. Le buffle ne se maintient, au Cambodge, que parce que les abatages de bufflesses y sont peu fréquents et que l'exportation des femelles étant interdite, toutes les possibilités de production y sont sauvegardées.

Conclusions. - A la suite de cette étude, on peut se permettre une estimation du cheptel cambodgien, estimation qui ne s'écarte guère du chiffre des recensements. Trait à noter : le nombre des animaux soumis à l'impôt (bceufs adultes, buffles et bufflesses adultes) correspond à celui des effectifs officiels, ce qui marque bien qu'en ce domaine la fiscalité est suffisamment précise et qu'il n'y a pas lieu de la pousser davantage.

\begin{tabular}{|c|c|c|c|c|}
\hline & BEUFS & $\%$ & BUFFLES & $\%$ \\
\hline Mâles adultes . & 400.000 & $35 \%$ & 130.000 & $35 \%$ \\
\hline Femelles adultes. & 300.000 & $25 \%$ & 110.000 & $27 \%$ \\
\hline $\begin{array}{r}\text { Jeunes produits } \\
\text { de } 1 \text { à } 3 \text { ans.. }\end{array}$ & 300.000 & $25 \%$ & 100.000 & $25 \%$ \\
\hline $\begin{array}{l}\text { Sujets du premier } \\
\text { âge } \ldots \ldots \ldots \ldots\end{array}$ & 160.000 & $15 \%$ & 50.000 & $13 \%$ \\
\hline Total. & 1.160 .000 & & 390.000 & \\
\hline
\end{tabular}

La fertilité du troupeau doit être considérée comme bonne avec $72 \%$ chez la vache zébu et $61 \%$ chez la bufflesse. Cependant, le taux d'accroissement reste faible en raison d'une mortalité excessive dans le premier âge. Tout porte à croire que l'accroissement actuel est de l'ordre de $5 \%$ sur le troupeau de vaches et de bufflesses, chiffre très 
faible, si on le compare avec celui observé en d'autres pays.

L'abatage annuel de 20.000 génisses âgées de 2 à 4 ans coûte plús cher au troupeau bovin que les dévastations des épizooties. Les défenses édictées contre ces massacres restent inefficaces. Par la suppression de ces abatages, le troupeau bovin pourrait théoriquement doubler en moins de dix ans. On y trouverait également un àantage appréciable. le rajeunissement du troupeau par substitution plus complète des génisses de 3 ans aux vaches les plus anciennes. Selon nos estimations, il existcrait environ 45.000 vaches d'âge avancé qu'il y aurait intérêt à remplacer par des vaches plus jeunes dont la production est plus régulière. Les réductions pratiquées sur les vaches âgées permettraient de substituer des quantités de viande supérieures à celles provenant des destructions de génisses. Il faudra; à cet égard, transformer la mentalité du paysan, l'amener à un plus juste souci de ses intérêts et combattre l'attendrissement qui' lui fait épargner une vache âgée, parce que suitée, alors qu'il est insensible à l'abatage d'une génisse.

Le troupeau bubalin est moins bien pourvu et son accroissement, qui reste très faible, est périodiquemént entravé par le développement des enzooties do pcste bovinc, l'espèce y étant très sensible.

De cette analyse, aussi scrupuleuse que le permet la dangereuse précision des chiffres, il faut retenir que le cheptel cambodgien est au point mort. 